\title{
VELOCITY BUNCHING AT THE EUROPEAN XFEL
}

\author{
B. Beutner, W. Decking, M. Dohlus, K. Flöttmann, M. Krasilnikov, T. Limberg \\ DESY, Germany
}

\section{Abstract}

This paper explores the possibility to employ velocity bunching [1] in the first RF module of the European XFEL [2] (referred to as XFEL in this paper) to increase the peak current at the injector exit. The current increase will reduce the total longitudinal bunch compression factor and loosen rf jitter tolerances by the same amount. The relation between rf tolerances and micro-bunching instability gain is discussed and the injector optimization for cases of velocity bunching to $100 \mathrm{~A}$ and $200 \mathrm{~A}$ peak current are presented in detail.

Finally, plans for velocity bunching experiments at the FLASH facility (Free Electron Laser in Hamburg) [3] are laid out.

\section{INTRODUCTION}

\section{Principle}

Fig. 1 shows the velocity bunching scheme as it is applied in simulation calculations for FLASH and the XFEL.

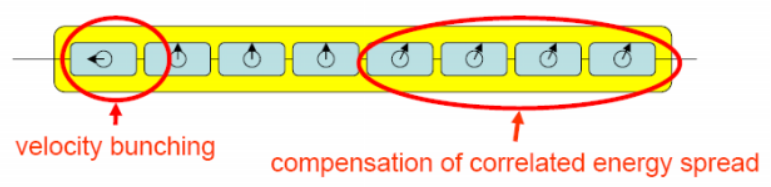

Figure 1: Velocity Bunching Scheme

The first cavity in a TESLA module is operated close to the zero-crossing phase and the resulting velocity differences along the bunch provide longitudinal compression. The induced correlated energy spread can be partly (FLASH) or fully (XFEL) taken out by running off-crest in the last four cavities.

\section{RF Tolerances and Micro-bunching Instability}

The optimization of a bunch compression system has to take into account the jitter tolerances for amplitudes and phases of the different involved rf systems as well as the strength of the micro-bunching instability.

We developed a tool which scans possible working points for the bunch compression system, varying the strength of the magnetic chicanes and the rf induced energy chirps, and calculates the above quantities.

It turns out that optimization for loose rf tolerances mostly leads to a stronger micro-bunching instability and, respectively, vice versa (see Fig. 2).

Velocity bunching reduces the bunch length at the entrance of the magnetic bunch compression system. The rf tolerances - which depend on the total compression factor in the magnet chicanes - are loosened by the same factor by which the beam is shortened by velocity bunching.

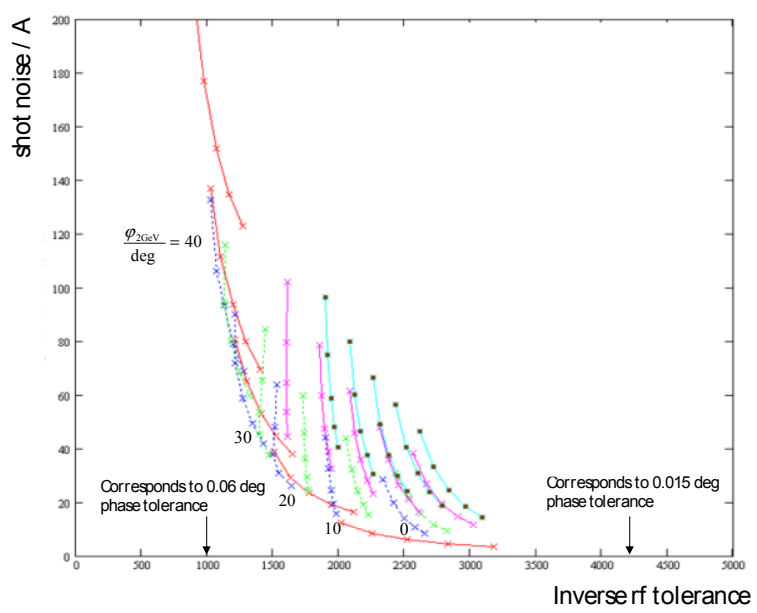

Figure 2: Shot noise in the undulator bunch profile (rms) and the inverse of the tightest rf tolerance for different bunch compression system working points. All points deliver a 5kA bunch, employing different $\mathrm{rf}$ phases and magnetic chicane strengths.

The gain of the micro-bunching instability is also reduced if the uncorrelated energy spread in the undulator is kept constant. The reduced compression factor in the magnet chicanes allows a higher laser-heater induced uncorrelated energy spread and a more effective damping of the instability [4].

\section{INJECTOR OPTIMIZATION}

\section{Nominal Case}

The photo injector for the XFEL consists of an 1.5-cell rf gun, operated in the pi-mode at $1.3 \mathrm{GHz}$, with solenoid magnets for beam focusing and projected emittance compensation. For nominal settings [2] it should provide a projected emittance below $0.9 \mathrm{~mm}-\mathrm{mrad}$ at a $1 \mathrm{nC}$ bunch charge. A flat-top temporal profile with 20 ps fwhm and 2 ps rise/fall time is used for the photo cathode laser. After emission from the Cs2Te photo cathode the electron bunch is accelerated by the rf field of the gun cavity. In order to suppress space charge induced emittance growth a high gradient will be applied (60MV/m at the cathode).

The main rf gun parameters for the optimization are the transverse spot size of the laser at the cathode, the rf launch phase and the main solenoid peak field. 
The electron bunch from the rf gun is injected into the first accelerating module (ACC1), which consists of 8 TESLA cavities. For the nominal settings of ACC1, the rf gradients and phases in the first and last four cavities will be tuned independently. The longitudinal position of ACC1 is also optimized.

For the injector optimization a staged strategy has been used: first, the gun parameters (laser spot size, launch rf phase and main solenoid peak current) have been varied, then for the chosen rf gun parameter set the first accelerating module has been tuned (its z-position, gradients and phases) in order to minimize the normalized projected emittance after ACC1. Beam parameter development along the beam line is shown in Fig.3. For the nominal setup an rms bunch length of $2 \mathrm{~mm}$ has been achieved at the injector exit, corresponding to a peak current of $\sim 50 \mathrm{~A}$.

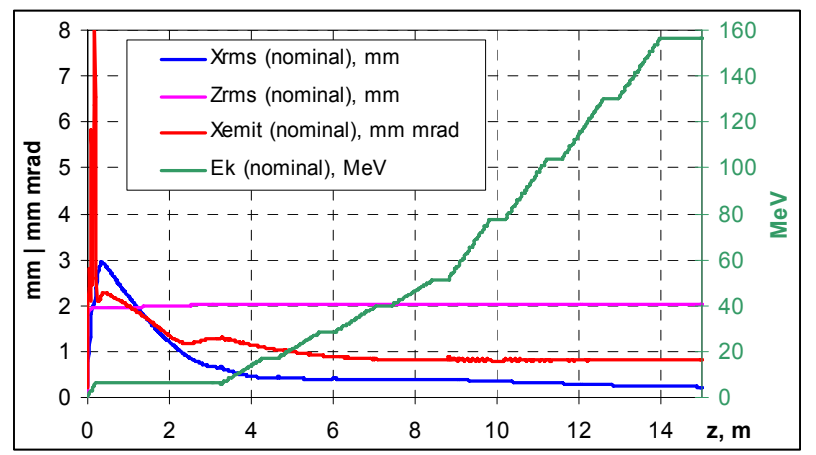

Figure 3: Electron beam parameters for the nominal setup: rms transverse size (Xrms), bunch length (Zrms), normalized transverse projected emittance (Xemit). The mean kinetic energy (Ek) of the bunch is plotted on the right axis.

\section{Velocity Bunching Settings}

For the beam dynamics simulations of the velocity bunching settings the longitudinal positions of the gun solenoid and ACC1 were kept at nominal values.

A peak current of $100 \mathrm{~A}$ has been chosen as a goal for the first optimization. The phase of the first cavity in ACC1 is set strongly off crest to produce a chirp in the longitudinal phase space. This implies no acceleration in this cavity and therefore, in principle, the emittance increases due to space charge effect. The nominal matching conditions for the emittance conservation are not valid anymore. To reduce the emittance growth, the main solenoid peak field has been tuned.

For a peak beam current of $200 \mathrm{~A}$, a more extensive optimization, involving tuning of the laser spot size at the cathode and running the fifth cavity in the ACC1 strongly off crest (i.e. to use it as the second bunching cavity), has been done.

Main parameters for the different settings are summarized in Table 1.
Table 1: Main parameters

\begin{tabular}{|l|c|c|c|}
\hline Parameter & Nominal & $\begin{array}{c}\text { Case } \\
\mathbf{1 0 0} \text { A }\end{array}$ & $\begin{array}{l}\text { Case } \\
\mathbf{2 0 0} \text { A }\end{array}$ \\
\hline $\begin{array}{l}\text { rms Laser spot size at cathode, } \\
\text { mm }\end{array}$ & 0.44 & 0.44 & 0.57 \\
\hline Main solenoid peak field, T & 0.224 & 0.218 & 0.218 \\
\hline ACC1 cavity 1 phase, deg & -15.7 & -98 & -102.2 \\
\hline ACC1 cavities 2-4 phase, deg & -15.7 & -15.7 & 14.2 \\
\hline ACC1 cavity 5 phase, deg & -15.7 & -15.7 & -79.8 \\
\hline ACC1 cavities 6-8 phase, deg & -15.7 & -15.7 & 14.2 \\
\hline
\end{tabular}

The results of the optimization are shown in Fig.4.

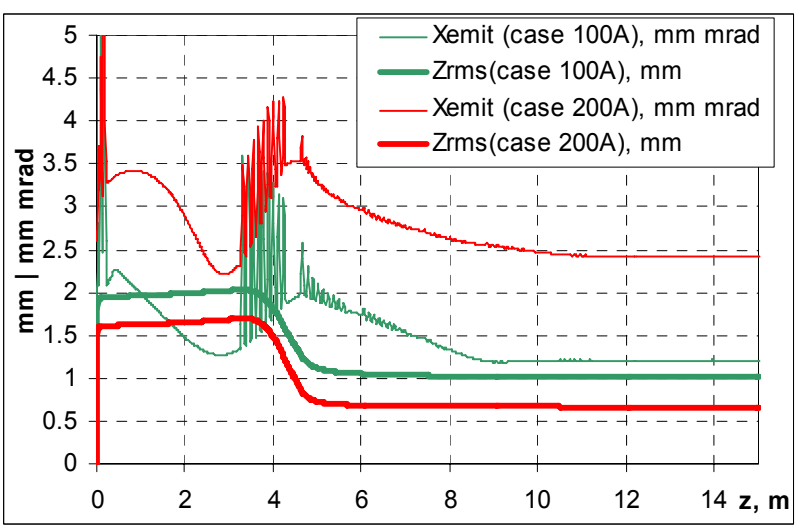

Figure 4: Electron beam parameters for the velocity bunching options: rms transverse projected emittance (Xemit) and bunch length (Zrms).

The slice transverse emittance of the electron bunch at $\mathrm{z}=15 \mathrm{~m}$ is shown in Fig. 5 together with corresponding beam current profiles.

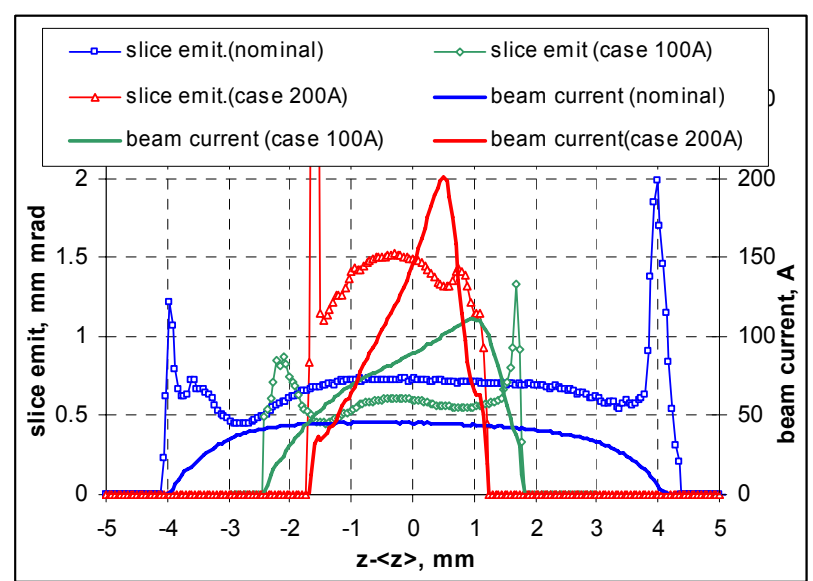

Figure 5: Slice emittance and beam current profiles of electron bunch at $\mathrm{z}=15 \mathrm{~m}$.

It should be noticed that the local slice emittance for the $100 \mathrm{~A}$ case is even lower than in the nominal case, whereas the projected emittance is higher $(1.2 \mathrm{~mm} \mathrm{mrad})$ than in the nominal case $(0.8 \mathrm{~mm} \mathrm{mrad})$. This is due to the 
local transverse phase space correlations along the bunch. For the nominal case, local phase space ellipses are almost perfectly aligned. In the $100 \mathrm{~A}$ case, phase space ellipses (although having locally smaller areas) are misaligned along the bunch.

The slice energy spread is shown in Fig.6.

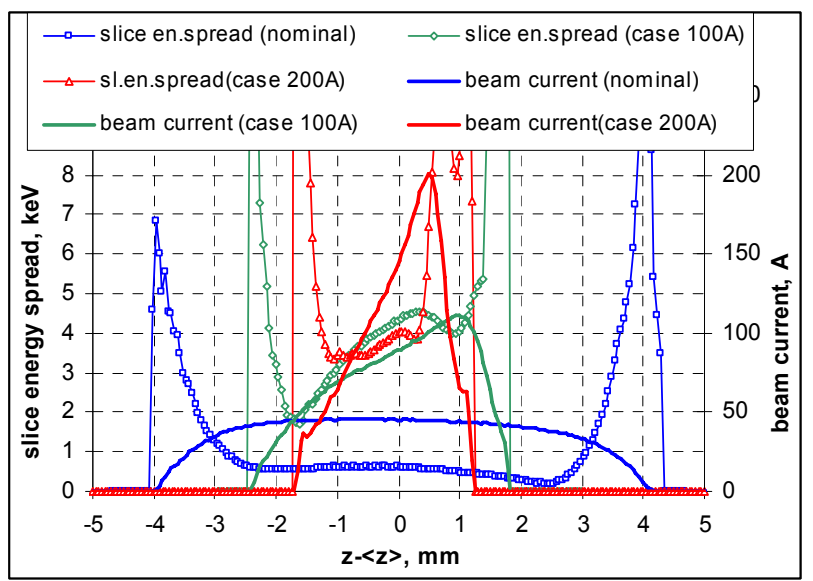

Figure 6: Slice energy spread and beam current profiles of electron bunch at $\mathrm{z}=15 \mathrm{~m}$.

\section{VELOCITY BUNCHING STUDIES AT FLASH}

Experimental velocity bunching studies at FLASH are a prerequisite for using it in the XFEL. As described earlier the first cavity in $\mathrm{ACC} 1$ is set up to operate around the zero crossing of the field. The evolution of bunch length through ACC1 is shown in Fig. 7.

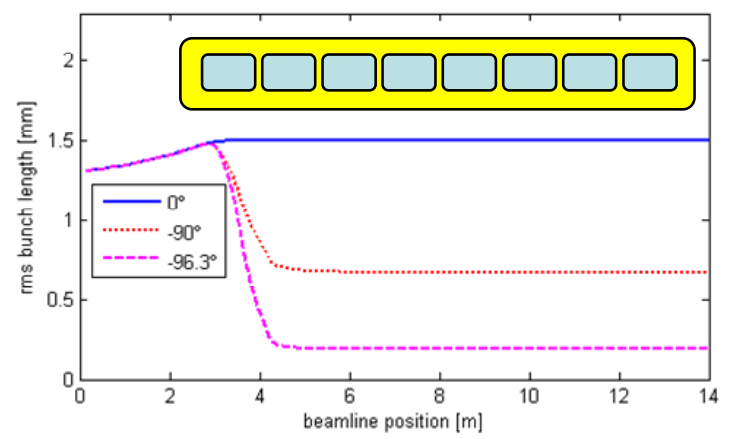

Figure 7: Bunch length along ACC1 for different phase offsets in the first cavity. No compression for on-crest acceleration, full compression at -96.3 degrees, and medium compression (keeping space charge emittance growth tolerable) at 90 degrees.

The rms bunch length and the peak current as a function of the phase offset in the first cavity of ACC1 are presented in Fig. 8. The main injector parameters for these simulations are taken from measurements at FLASH. The cathode gradient is $44 \mathrm{MV} / \mathrm{m}$ and the accelerating gradients in the first four cavities are 13.0 $\mathrm{MV} / \mathrm{m}, 13.5 \mathrm{MV} / \mathrm{m}, 10.5 \mathrm{MV} / \mathrm{m}$, and $12.8 \mathrm{MV} / \mathrm{m}$. The last four cavities of ACC1 have a gradient of $17.6 \mathrm{MV} / \mathrm{m}$.
The beam enters the first cavity wit a total energy of $\sim 4.5 \mathrm{MeV}$ and exits ACC1 with $134 \mathrm{MeV}$. Bunch charge for the calculations is $1 \mathrm{nC}$.

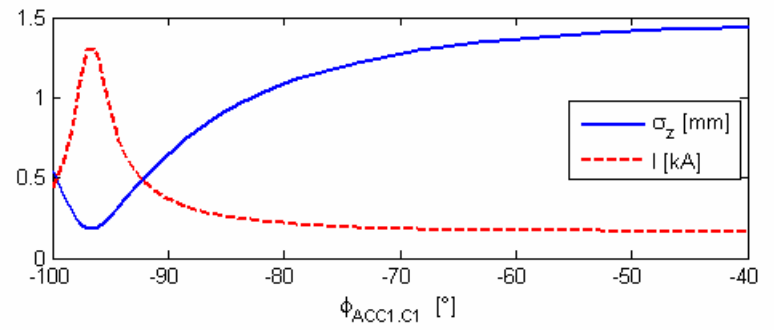

Figure 8: ASTRA [5] simulation of the rms bunch length and peak current the exit of $\operatorname{ACC} 1(\mathrm{z}=14.0 \mathrm{~m})$ versus the phase of the first cavity of ACC1.

At FLASH measurements of the emittance and the bunch length versus the phase offset in the first cavity are prepared for autumn 2007. OTR screens in a FODO section downstream of $\mathrm{BC} 2$ (see Fig. 9) are used for the emittance measurements. A streak camera at the synchrotron radiation port of the magnetic chicane $\mathrm{BC} 2$, and a transverse deflecting structure downstream of ACC5 are foreseen for the bunch length measurement.

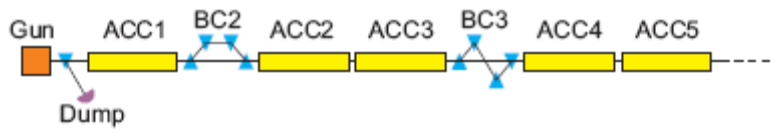

Figure 9: Sketch of the FLASH Injector

\section{SUMMARY AND OUTLOOK}

At the XFEL, velocity bunching could be useful to increase the peak current of the injector (gun and ACC1) from about 50A to maximal $200 \mathrm{~A}$, where the slice emittance equals the design specification for the whole accelerator. Start-to-End calculations still have to be performed to study the subsequent bunch compression in the magnetic chicanes. Machine experiments at FLASH are foreseen this fall.

\section{REFERENCES}

[1] L. Serafini and M. Ferrario, "Velocity Bunching in Photo-Injectors", AIP conference proceedings 581, p.87-106, 2001

[2] A. Aghababyan et al., "The European X-Ray FreeElectron Laser Technical Design Report". DESY XFEL Project Group http://xfel.desy.de/tdr/index_eng.html

[3] W. Ackerman et al., "Operation of a free electron laser from the extreme ultraviolet to the water window", Nature Photonics, vol.1, 2007, pp. 336342.

[4] W. Decking and T. Limberg: "Beam Dynamics at FLASH and the European XFEL" ICFA Newsletter 42, p.34-45

[5] K. Floettmann, ASTRA User Manual, see http://www.desy.de/ mpyflo/Astra_dokumentation/. 